\title{
Dynamic Hip Screw Fixation of Intertrochanteric Fractures of Femur: A Comparison of Outcome With and Without Using Traction Table
}

\author{
SG Gooi, MD (USM), EH Khoo, MS Orth, Benny Ewe, MBBS, Yacoob, MS Orth \\ Department of Orthopaedic and Traumalogy, Hospital Seberang Jaya, Pulau Pinang, Malaysia
}

\begin{abstract}
Intertrochanteric fracture of the femur is one of the common fractures in the elderly. Dynamic Hip Screw (DHS) fixation is the gold standard for treatment of intertrochanteric femoral fracture. Conventional methods of achieving reduction prior to instrumentation require utilization of a traction table. A manual traction technique applied in the supine position using only a translucent table was devised to do away with the use of traction table. The rationale for this technique includes enhanced ease of set up, the ability to perform multiple procedures without repositioning and prevention of traction table related complications. Use of the reduction method with a traction table or application of manual traction during dynamic hip screw fixation of intertrochanteric femoral fracture was both feasible and safe.
\end{abstract}

Key Words:

Dynamic Hip Screw, Traction Table, Intertrochanteric Hip Fracture

\section{INTRODUCTION}

Intertrochanteric hip fracture is a common injury treated by orthopaedic surgeons. A variety of hip fracture fixation devices are available for treatment of intertrochanteric fracture, and dynamic hip screw (DHS) has been the gold standard treatment of intertrochanteric fracture of femur ${ }^{1-2}$. DHS is based on the concept of allowing fracture fragments to impact, thereby achieving bone-on-bone stability, and reducing chances of implant failure, so called "controlled collapse". Such collapses continue until proximal fragment rests on, stable, intact distal fragments.

Traction tables are presently used universally as a standard tool for DHS fixation to achieve and maintain satisfactory reduction before instrumentation is performed. However, a significant amount of time is necessary for preoperative set up when a traction table is to be used. Although there have not been many reports on traction table associated complications during DHS fixation, problems such as pudendal, sciatic or femoral nerve injury, due to traction or direct pressure are not uncommon in patients undergoing femoral nailing, as it involves a large amount of traction force $^{3-5}$. Furthermore, a traction table may not be available in hospitals with limited resources. Hence, a manual traction technique performed in the supine position using only a radiolucent table was devised to do away with the necessity of using a traction table. The rationale for not using the traction table includes ease of set up, the ability to perform multiple procedures with a single positioning and draping, and the elimination of morbidities associated with use of traction table. The objectives of this study were to assess the feasibility of performing intertrochanteric hip fracture reduction and DHS fixation without using a traction table. Feasibility was assessed for several technical aspects of the technique. The quality of fixation was assessed by comparing the Tip Apex Distance (TAD) measured in postoperative radiographs. Clinical outcomes from other techniques are also compared. Post-operative complications associated with these techniques such as shortening, malalignment and cut-out rate were reported.

\section{MATERIALS AND METHODS}

This is a retrospective comparison study involving all patients admitted to Hospital Seberang Jaya with intertrochanteric fractures of the femur who were treated with DHS from March 2008 to February 2009. Exclusion criteria include pathological fractures, patient age younger than 16 years, and unstable 3 or 4 part intertrochanteric fractures. Based on the technique of reduction during DHS fixation, the patients were divided into two groups. Patients who underwent reduction using a traction table were recruited into Group 1 while Group 2 patients were reduced with manual traction and manipulation without utilization of a traction table. All of the patients were treated with skin traction after admission to the ward but prior to the surgical procedure. The surgical procedures were explained, written informed consent was obtained from all patients and patients were scheduled for DHS. All procedures were performed under regional anaesthesia. 
Table I: Statistical analysis of various parameters in 2 groups of patients

\begin{tabular}{|lccc|}
\hline Parameter & $\begin{array}{c}\text { Mean } \\
\text { Group 1 }\end{array}$ & Group 2 & P value \\
\hline TAD & 20.7 & 23.1 & 0.841 \\
Difference in & & & \\
Neck-Shaft Angle & 6.5 & 7.7 & 0.557 \\
Blood loss & 151 & 166 & 0.815 \\
Union rate & $13.4 \mathrm{w}$ & $12.7 \mathrm{w}$ & 0.297 \\
Duration of surgery & 57 mins & $54.6 \mathrm{mins}$ & 0.08 \\
Pre-operative & 31mins & $14 \mathrm{mins}$ & 0.005 \\
preparatory time & & & \\
\hline
\end{tabular}

TAD : tip-apex distance

For Group 1 patients, the conventional reduction technique was performed utilizing a traction table under Imaging Intensifier (I.I.) guidance before instrumentation. Satisfactory reduction in both antero-posterior (AP) and lateral views was obtained. Then, cleaning and draping of the patient and the standard techniques for DHS instrumentation were performed. The reduction was maintained throughout the procedure without much manipulation intra-operatively. For Group 2, patients were placed on a radiolucent table in supine position in a frog leg view (in which the hip is flexed and abducted), and the affected lower limb was cleaned and draped. The positioning and draping of the patient took into consideration the position of the guide wire and other instruments as positioning was close to the edge of the table to prevent obstruction of the instruments by the table. A rolled towel was positioned underneath the ipsilateral buttock to facilitate instrumentation. Manual traction and reduction was performed under Imaging Intensifier guidance and an AP view of the fracture was obtained with the hip in supine position (Figure 1). One or two $3 \mathrm{~mm}$ Steinmann pins were then inserted to fix the preliminary reduction. The Steinmann pins were positioned in a fashion that avoided the intended guide wire position. A frog-leg lateral view of the fracture was then obtained by flexing and abducting the hip (Figure 2). If the reduction was satisfactory in the lateral view as well, the remainder of procedure would be almost identical to standard DHS instrumentation technique. However, if the reduction was not acceptable in the lateral view (i.e. with distal fragment displaced anteriorly or posteriorly), the Steinmann pins would be removed and reduction would be repeated until it was satisfactory in both the AP and lateral view.

For assessment of the reduction and fixation, post-operative radiographs were taken to measure the TAD and the difference in neck shaft angle compared to the contralateral hip. Statistical analysis was performed to assess differences between the two groups and differences were tested for statistical significance. Comparisons include the total operative time and pre-operative preparation time. Total operative time was defined as the duration of the surgery from skin incision to skin closure. Preoperative preparation time was defined as the time from anaesthesia induction to skin incision time, during which the positioning of patients, reduction under I.I. (Group 1) and draping was performed. Other study parameters include patient age, extent of fracture comminution, surgical times, estimated blood loss, length of hospitalization, union rate, time to clinical radiographic union, and complication rate. All of this information was obtained from medical records, radiographs, and patient interviews during follow-ups.

\section{RESULTS}

The total number of patients with intertrochanteric fracture recruited for this study was 40 patients. Group 1 consisted of 24 patients, while Group 2 consisted of 16 patients. All patients were followed up for an average of 46 weeks (range, 24 to 70 weeks). The mean patient age was 73 years old (range, 22 to 92y). There was no statistically significant difference in age between both groups. The pre-operative preparation time for Group 1 was 31 minutes and 14 minutes for Group 2, indicating a significantly shorter preoperative duration for the manual traction group $(\mathrm{p}<0.05)$. However, the average total surgical duration for Group 1 was 57 minutes and 54.6 minutes for Group 2, not a significant difference (Table I). In terms of fracture reduction and fixation, the TAD for Group 1 was 20.7 and 23.1 for Group 2 (not a statistically significant difference). For the post-operative neck-shaft angle, the difference to the contralateral hip in each group was compared. The difference between groups was only 1.2 degree, not significant (Table I). There was also no significant difference in blood loss, union rate, and time to clinical union (Table I). There was one implant cut out from each group, which was not significant statistically.

\section{DISCUSSION}

The practice of achieving diaphyseal femoral fracture reduction without a traction table for subsequent nailing procedures has been widely practiced and reported in the literature $^{6-9}$. However, the application of such techniques in intertrochanteric fracture of femur has not thoroughly analysed in the literature. We believe that reduction of intertrochanteric fracture of femur without a traction table is an indispensable option under certain circumstances mentioned above.

One of the biggest challenges in DHS instrumentation is to obtain satisfactory reduction in both AP and lateral views during the placement of the guide wire. In the conventional technique, both views can be obtained easily. The lateral view can be achieved after positioning the contralateral lower limb in a lithotomy position (not obstructing the Carm). In the manual traction technique on the other hand, the contralateral hip blocks the lateral view of the fracture site in supine position. Due to the proximity of both the hips, adjusting the $\mathrm{C}$-arm position alone does not produce a 


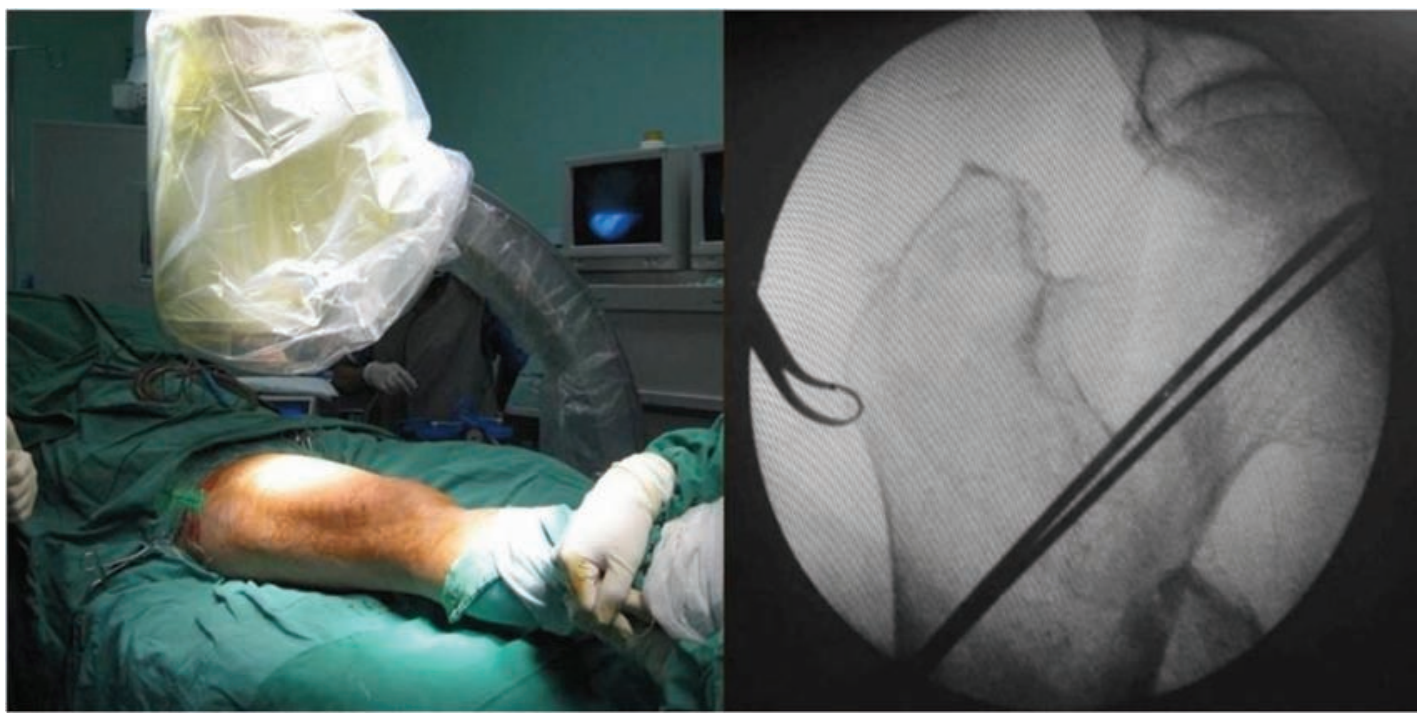

Fig. 1: Manual traction was applied in AP view under image intensifier examination before the guide wires are inserted.

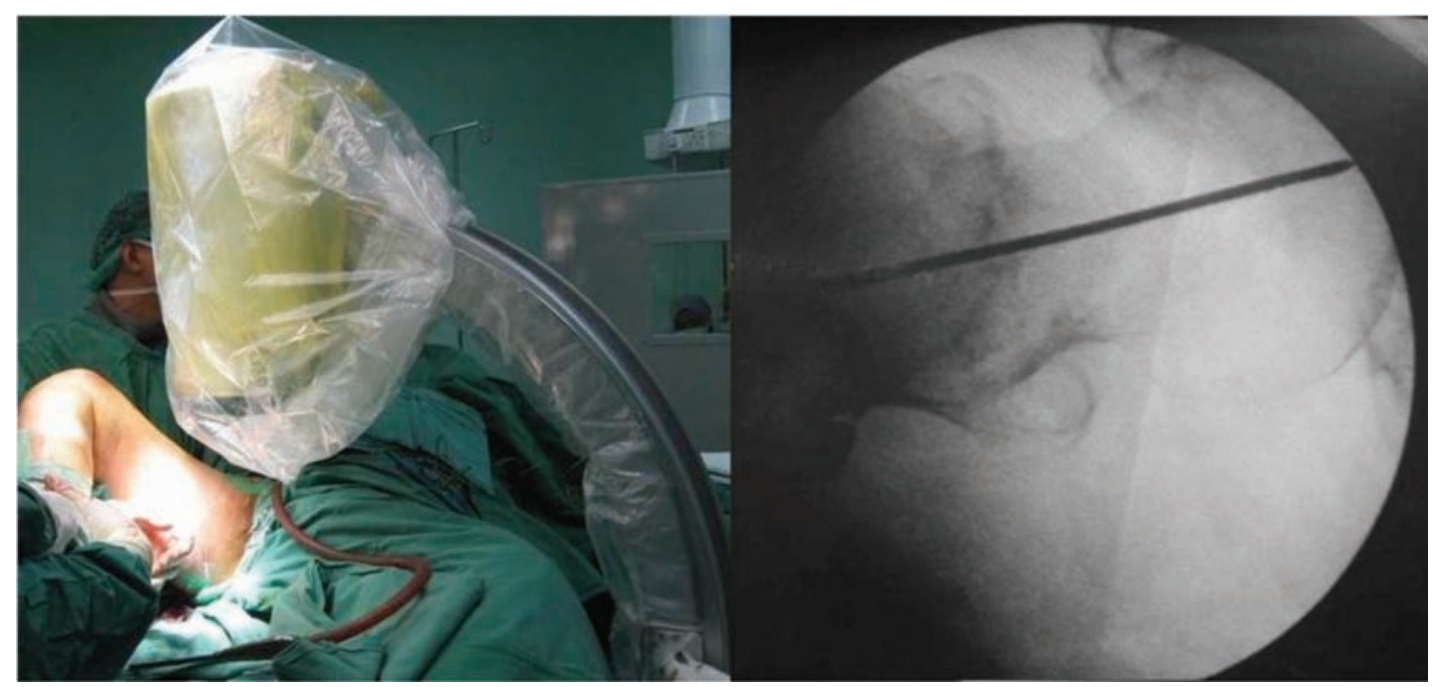

Fig. 2: Frog leg lateral view was obtained after preliminary fixation with guide wires.

satisfactory lateral view. This problem however can be overcome by positioning the affected lower limb in frog leg position and the $\mathrm{C}$-arm in vertical position. The lateral frogleg view has been advocated to obtain a satisfactory lateral view of the hip by arthroplasty surgeons ${ }^{12,13}$. Lateral views obtained in this position were "true" lateral views that were adequate to verify fracture reduction.

This technique however poses a few difficulties. During positioning the lower limb in frog leg position, the fracture site is subjected to a fair amount of stress. Although the fracture site should be adequately fixed with guide wires, frequently the stress exerted is too strong and may displace the reduction achieved beforehand. Our solution to this problem was to either to increase the number of temporary guide wires or $3 \mathrm{~mm}$ Steinmann pins to secure the temporary fixation.
A few technical points are worth mentioning for this technique. An important prerequisite is to obtain a satisfactory lateral view without jeopardizing the reduction; this requires a frog leg posture. Hence, an advanced arthritic hip that has reduced range of motion may not be a suitable candidate for this technique, since satisfactory frog-leg view may be impossible to achieve. We noticed on most occasions that the Steinmann pin would be bent slightly during lateral view I.I. examination. This was probably due to a deforming force while trying to position the hip in frog leg position. Although a slight bend will not jeopardize the reduction, significant bends were seen occasionally. We recommend that the few crucial steps, for example, the advancement of the guide wire and the reaming of the core screw tract, should be performed only when the lower limb is in neutral position. The above steps should not be performed when the limb is in frog-leg position, meaning that this view should only be used for purposes of checking reduction. Subsequent 
reaming over a bent guide wire will cause breakage of the wire.

To evaluate and compare the quality of the fixation, TAD was used ${ }^{12-14}$. In our sample, the difference in the TAD score in both groups was not statistically significant. This points to a comparable effectiveness of fixation for both techniques. Group 1 has a slightly better score, though not statistically significant, which was probably due to the slight advantage in reduction using a fracture table. When comparing both techniques, we note that utilization of a fracture table maintains reduction throughout the fixation procedure. Without a fracture table, the hip must be mobilized throughout the procedure to ensure continued maintenance of the reduction. Despite this issue, outcomes were not significantly inferior to the traction table technique.

Achieving reduction through manual traction and manipulation results in statistically significant shorter preoperative preparation time. This period was expected to be shorter than in the conventional technique, where more time was spent achieving reduction under fluoroscopic guidance. The theoretical time saved due to shorter preparation time could however be outweighed by the prolonged intraoperative fracture reduction time. The differences in timing are not clinically significant to the overall outcome.

A disadvantage of manual traction technique was the need of an extra assistant who had to maintain relatively constant traction to prevent displacement of the reduction. Using a Schantz screw with a T handle as a joystick was a 'trick' used to achieve desired reduction. In addition, more frequent I.I. images may be needed as preliminary reduction is achieved through a 'trial and error' procedure since lateral visualization was not possible before preliminary fixation with $3 \mathrm{~mm}$ Steinmann pin. This part of the manual traction technique is more technically demanding and results in a less predictable outcome.
Although no complications occurred that were attributable to the technique, there exist potential complications that should be avoided such as: broken wires or pins due to excessive manipulation when using frog leg view; fracture of the lateral cortex of the intertrochanteric region due to multiple drill holes; and, prolonged surgical time due to increased technical demand. Hence the standard traction table technique is still preferable in intertrochanteric fracture fixation in normal situations.

Other parameters measured revealed small differences that were not statistically significant. This includes rate of union, operative blood loss, and rates of post-operative complications. Limitations of this study include small sample size and the fact that it was a retrospective study. Further for consistency across all subjects, optimally a single surgeon should ideally perform all the procedures. Fluoroscopic time of both groups would be another important parameter for comparison that was not included in the present study.

\section{CONCLUSIONS}

Reduction and fixation of intertrochanteric fracture via manual traction is feasible and effective. It significantly reduces preoperative preparation time without sacrificing reduction alignment, screw position and bone healing. This technique is indispensable under circumstances where traction table is not available or frequent operative bed transfer should be avoided as in polytrauma patients where multiple procedures are necessary. 


\section{REFERENCES}

1. Larsson S, Elloy M, Hansson LI. Fixation of unstable trochanteric hip fractures. A cadaver study comparing three different devices. Acta Orthop Scand 1988; 59(6): 658-63.

2. Mulholland RC, Gunn DR. Sliding screw plate fixation of intertrochanteric femoral fractures. J Trauma. 1972; 12(7): 581-91.

3. Anglen J, Banovetz J. Compartment syndrome in the well leg resulting from fracture-table positioning. Clin Orthop Relat Res 1994; 301: 239-42.

4. Brumback RJ, Ellison TS, Molligan H, Molligan DJ, Mahaffey S, Schmidhauser C. Pudendal nerve palsy complicating intramedullary nailing of the femur. J Bone Joint Surg Am 1992; 74(10): 1450-5.

5. Callanan I, Choudhry V, Smith H. Perineal sloughing as a result of pressure necrosis from the traction post during prolonged bilateral femoral nailing. Injury 1994; 25(7): 472.

6. Aiyer S, Jagiasi J, Argekar H, Sharan S, Dasgupta B. Closed antegrade interlocked nailing of femoral shaft fractures operated up to 2 weeks post injury in the absence of a fracture table or C-arm. $J$ Trauma 2006; 61(2): 457-60.

7. Karpos PA, McFerran MA, Johnson KD. Intramedullary nailing of acute femoral shaft fractures using manual traction without a fracture table. J Orthop Trauma 1995; 9(1): 57-62.

8. Reynders P, Broos P. Unreamed intramedullary nailing of acute femoral shaft fractures using a traction device without fracture table. Acta Orthop Belg 1998; 64(2): 175-9.

9. Sirkin MS, Behrens F, McCracken K, Aurori K, Aurori B, Schenk R. Femoral nailing without a fracture table. Clin Orthop Relat Res 1996; 332: 119-25.

10. Clohisy JC, Nunley RM, Otto RJ, Schoenecker PL. The frog-leg lateral radiograph accurately visualized hip cam impingement abnormalities. Clin Orthop Relat Res 2007; 462: 115-21.

11. Konan S, Rayan F, Haddad FS. Is the frog lateral plain radiograph a reliable predictor of the alpha angle in femoroacetabular impingement? J Bone Joint Surg [Br] 2010; 92(1): 47-50.

12. Baumgaertner MR, Curtin SL, Lindskog DM, Keggi JM. The value of the tip-apex distance in predicting failure of fixation of peritrochanteric fractures of the hip. J Bone Joint Surg [Am] 1995; 77(7): 1058-64.

13. Baumgaertner MR, Solberg BD. Awareness of tip-apex distance reduces failure of fixation of trochanteric fractures of the hip. $J$ Bone Joint Surg [Br] 1997; 79(6): 969-71.

14. Cool P. The value of the tip-apex distance in predicting failure of fixation of peritrochanteric fractures of the hip. J Bone Joint Surg [Am] 1996; 78(9): 1447-8. 